\title{
Evaluating the moisture transfer of membranes for total heat exchanger
}

\author{
Nguyen Van $\mathrm{Vu}^{\mathrm{a}}$ and Václav Dvořák \\ Department of Power Engineering Equipment, Faculty of Mechanical engineering, Technical University of Liberec, \\ Czech Republic
}

\begin{abstract}
This paper presents an experimental evaluation of moisture transfer of a novel membrane which is used in enthalpy heat exchanger. The moister transfer through a membrane was investigated in a model of counter flow heat exchanger and the effectiveness of moister transfer was evaluated for various flow velocities. It was found that the measuring error, i.e. difference between moister absorbed and released is quite high. Therefore, measuring uncertainty analyses were performed. The resulting uncertainty of effectiveness of moister transfer was derived using partial derivatives of all influencing quantities and considering both random and systematic uncertainties. It was found that measuring of mass flow rate has the biggest influence on resulting uncertainty of moister transfer effectiveness. Therefore, accuracy of measured air flow rates measuring must be improved to reach lower resulting uncertainty.
\end{abstract}

\section{Introduction}

Air ventilation is important to maintain healthy condition for residential building, especially in cold climate areas. However, ventilation causes a huge energy lost, which has to be compensated by heating. Therefore, heat exchangers are used to get back part of heat energy from exhaust air and convey it to the fresh air. In the last decades, researchers focused on conventional heat exchangers which can recover only sensible heat. On the demand of increasing indoor air quality and decreasing energy consumed by the ventilation system, total heat exchanger (or enthalpy heat exchanger) has been investigated because of the following advantages: Total heat exchanger can recover not only sensible but also latent heat from exhaust air which means higher potential use. Moreover, by using enthalpy heat exchanger, we can avoid or reduce condensation and frost inside a heat exchanger, which is one of the most important disadvantages of aluminium or plastic based conventional heat exchangers when working at temperatures lower than dew point or freezing point temperature, respectively [1], [2]. Among several types of total heat exchangers, membrane-based enthalpy heat exchangers have great potential thanks to compactness and lightweight.

Due to the complex coupling between heat and moisture transfer, research and development of membranes for membrane-based heat exchanger is still in an early state and need more effort from researchers. Huge number of mathematical and numerical models were conducted to estimate effectiveness of heat and moisture transfer, pressure drop and so on, such as in works [2]-[5]. Many researches also focus on experimental method to evaluate prototype models or novel membranes, such as [1], [6]-[8]. Many investigations have been done, but most of researchers focused on their own configurations, there were no general model due to complex combinations of different factors.

As continuation of preliminary work [6], this paper presents evaluation of moisture transfer of novel membranes and a decent analysis of uncertainty in calculating moisture transfer effectiveness.

\section{Moisture transfer}

Structure of membranes prevents transfer of dust or polluted air while enable molecules of water to pass through. There are several driving forces, which cause the transfer of moisture though membrane, such as the difference of density and pressure between both sides of the membrane.

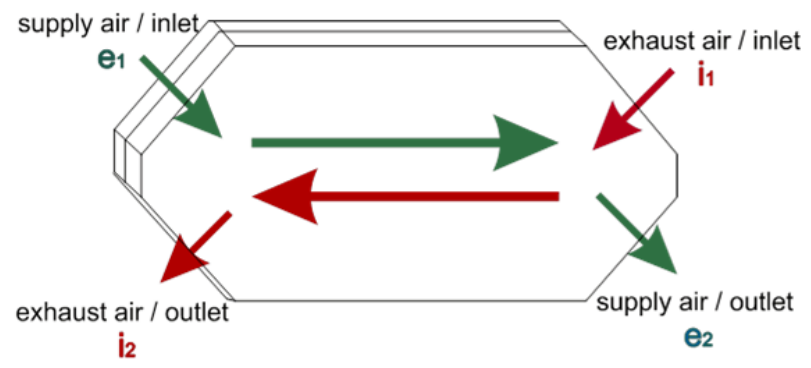

Figure 1: Counter flow plate heat exchanger.

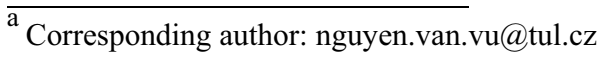




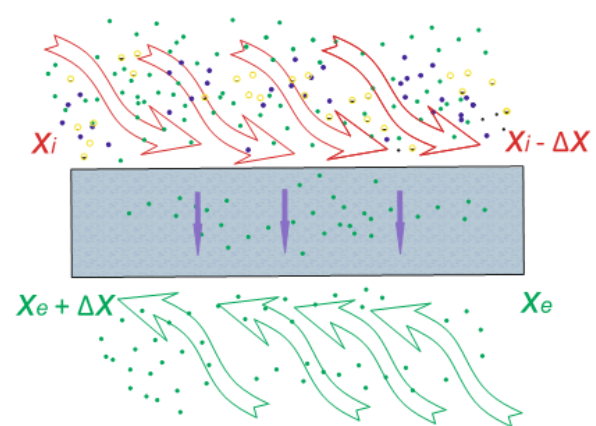

Figure 2: Illustration of moisture transfer through membrane.

Membrane separates the exhaust and supplied flow, prevents dust, bacterium and poisonous substances like $\mathrm{CO}, \mathrm{CO}_{2}, \mathrm{CH}_{4}$ and so on, meanwhile it allows moisture from exhaust air pass through to supplied air.

A specific experimental enthalpy exchanger for testing moisture transfer through membrane was build. Due to the real-life working condition of the enthalpy exchanger, we consider the driving force comes only from the density's difference. Therefore, we tried to ensure temperature, pressure of both flows (dry and moist airflow) are equal to each other during the whole measurement.

Psychometric diagram (figure 3) shows amount of specific humidity $\Delta x$ resp. specific enthalpy of the supply $\Delta h$ and the exhaust air, which are transferred to supply air flow. Their relation is given by equation

$$
\Delta h_{w}=\Delta x\left(l_{0}+c_{w} t\right)
$$

where $l_{0}$ is specific latent heat of vaporization

$$
l_{0}=2260 \mathrm{~kJ} / \mathrm{kg} \text { while } c_{w}=4180 \mathrm{~J} \cdot \mathrm{kg}^{-1} \cdot \mathrm{K}^{-1} \text { is }
$$

the specific heat capacity of water. By comparing terms in equation (1), it can be rewritten as

$$
\Delta h_{w}=\Delta x l_{0} \text {. }
$$

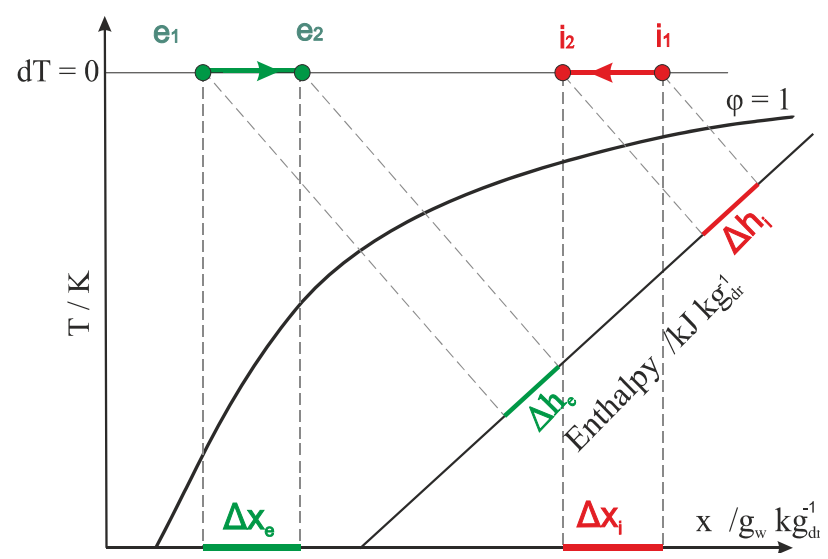

Figure 3: Illustration of recovered latent heat in psychometric diagram.

\section{Experimental arrangement}

The figure 4 describes experimental arrangement. Dry compressed air flows to T-junction (4) where it is divided into two ways. One of them is humidified by flowing through a vaporizer (7). By doing that, two levels of humidified air were created: high humidity of average around $75 \%$ and low humidity of averaged around $60 \%$. Volumetric flows of humidified and dry airs are controlled by valves (6) and then flow into experimental enthalpy exchanger, where they flow in counter flow arrangement but separated from each other by novel membrane (11). Volumetric flows were set from 200 litres per hour to 2000 litres per hour which correspond to typical flow velocity of $(0.26 \div 2.58) \mathrm{m} / \mathrm{s}$. We used high precision FHAD36R sensors to measure relative

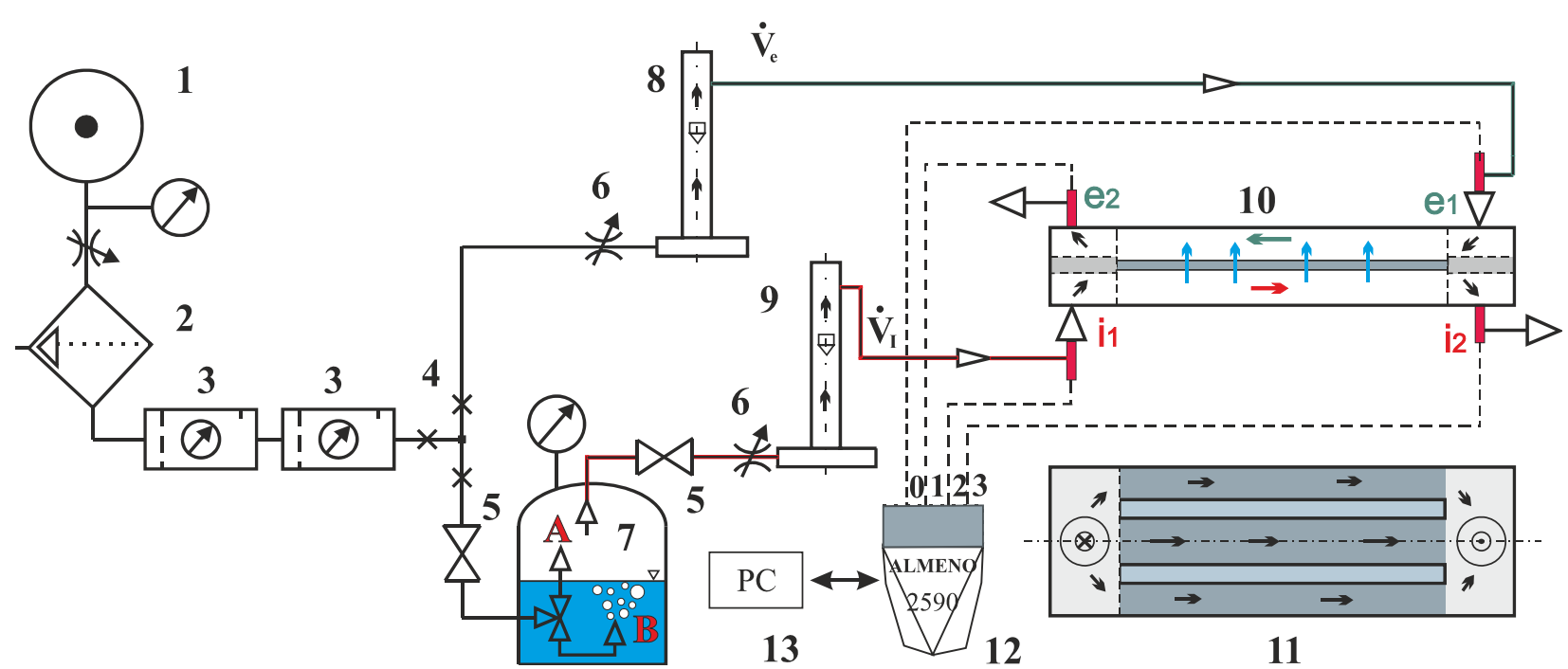

Figure 4: Experimental arrangement: 1 - compressor, 2 - air filter, 3 - valves, 4 - T-conjunction, 5 - flap, 6 - smooth valves, 7 - vaporizer, $8 \& 9$ - rotameters, 10 - experimental enthalpy exchanger, 11 - novel membrane, 12 - Almeno 2590, 12.0 - sensor for dry airflow inlet, 12.1 - sensor for dry airflow outlet , 12.2 - sensor for humidified-inlet airflow, 12.3 - sensor for humidifiedoutlet airflow, 13 - PC 
humidity, temperature, barometric pressure of dry and humidified airflows before and after moisture transfer through novel membrane. Device Almeno 2590 was used to $\log$ these values to PC for every second during measurement.

\section{Effectiveness of moisture transfer}

From the equation (2), effectiveness of latent heat transfer could be defined from [9] as

$$
\eta_{x}=\frac{x_{e 2}-x_{e 1}}{x_{i 1}-x_{e 1}}
$$

where $x$ is specific humidity defined as

$$
x=\frac{m_{\text {moisture }}}{m_{\text {dry air }}} .
$$

For calculation specific humidity from relative humidity, equation below is usually used [9]

$$
x=0.622 \frac{\varphi p_{p}{ }^{\prime}}{p_{b}+\varphi p_{p}}
$$

where $p^{\prime \prime}{ }_{p}$ is the partial pressure of saturated vapor calculated by

$$
p_{p}^{\prime \prime}=e^{23.58-\frac{4044.2}{235.6+t}}
$$

in which $t$ is temperature of air flow.

Process of moisture transfer through membrane is a sequence of two "sub-process". Firstly, absorption moisture of membrane from exhaust air with more humidity and secondly, moisture release from membrane to supply, dryer air. Relative measuring error of effectiveness of moisture transfer is given by ratio of difference of transferred humidity from both sides of membrane, i.e. difference between absorption and release, and average transferred humidity. For equal mass flow rates of dry air, the relative measuring error of effectiveness of moisture transfer is defined by ratio

$$
\varepsilon_{x}=2 \frac{\left|\Delta \mathrm{x}_{e}+\Delta x_{i}\right|}{\left|\Delta \mathrm{x}_{e}\right|+\left|\Delta \mathrm{x}_{i}\right|} \text {. }
$$

\section{Uncertainty}

The uncertainties of experimental measurements are connected to the accuracy, reliability of measuring instruments and the way they are manipulated. Uncertainty of every measuring device is defined as function of two values: random uncertainty and systematic uncertainty.

Standard deviation is defined as

$$
s=\sqrt{\frac{\sum(x-\bar{x})^{2}}{(n-1)}},
$$

where $\bar{x}$ is the mean value and $\mathrm{n}$ is the number of measurements

Random uncertainty $\delta_{r}$ (also known as uncertainty type A) is caused by unknown reasons and can be calculated from standard deviation. It means that random uncertainty follows from measured data as

$$
\delta_{r}= \pm \sqrt{\frac{\sum(x-\bar{x})^{2}}{(n-1) n}}= \pm \frac{s}{\sqrt{n}}
$$

The systematic uncertainty $\delta_{S}$ (also known as uncertainty type B) relates to several factors, first is given by the manufacturer and secondly by calibrations. The systematic uncertainty is specified by the manufacturer of the measuring device.

The total uncertainty then is

$$
\delta_{T}= \pm \sqrt{\delta_{r}^{2}+\delta_{S}^{2}}
$$

If quantity $A$ is not measured directly, but it is a function of some other quantities $\mathrm{f}\left(\mathrm{x}_{\mathrm{i}}\right)$, then uncertainty of quantity $A$ is according work [10]

$$
\delta_{\mathrm{A}}=\sqrt{\sum\left(\frac{\partial \mathrm{f}\left(\mathrm{x}_{i}\right)}{\partial \mathrm{x}_{i}} \delta x_{i}\right)^{2}}
$$

where $\frac{\partial \mathrm{f}\left(\mathrm{x}_{i}\right)}{\partial \mathrm{x}_{i}}$ is partial derivation of quantity $\mathrm{x}_{i}$ and $\delta x_{i}$ is the uncertainty of $x_{i}$. In following text, we used these formulas to specify uncertainty of effectiveness of moisture transfer.

Effectiveness of moisture transfer is defined as a ratio of actually transferred (released) humidity into dryer air and maximum theoretical humidity, which can be absorbed from moister air

$$
\eta=\frac{\dot{\mathrm{m}}_{e}\left(x_{\mathrm{e} 2}-\mathrm{x}_{\mathrm{e} 1}\right)}{\dot{\mathrm{m}}_{\iota}\left(\mathrm{x}_{\mathrm{i} 1}-\mathrm{x}_{\mathrm{e} 1}\right)} .
$$


The uncertainty of effectiveness is calculated

$$
=\sqrt{\begin{array}{c}
\left.\delta_{\eta} \frac{\partial \eta}{\partial \dot{\mathrm{m}}_{e}} \delta_{\dot{\mathrm{m}}_{e}}\right)^{2}+\left(\frac{\partial \eta}{\partial \dot{\mathrm{m}}_{i}} \delta_{\dot{\mathrm{m}}_{i}}\right)^{2}+\left(\frac{\partial \eta}{\partial \mathrm{x}_{\mathrm{i} 1}} \delta_{\mathrm{x}_{i 1}}\right)^{2}+ \\
+\left(\frac{\partial \eta}{\partial \mathrm{x}_{\mathrm{e} 1}} \delta_{\mathrm{x}_{e 1}}\right)^{2}+\left(\frac{\partial \eta}{\partial \mathrm{x}_{\mathrm{e} 2}} \delta_{\mathrm{x}_{e 2}}\right)^{2}
\end{array}}
$$

where

$\frac{\partial \eta}{\partial \dot{\mathrm{m}}_{e}}=\frac{\left(x_{e 2}-x_{e 1}\right)}{\dot{\mathrm{m}}_{i}\left(x_{i 1}-x_{e 1}\right)}, \frac{\partial \eta}{\partial \dot{\mathrm{m}}_{i}}=-\frac{\dot{\mathrm{m}}_{e}\left(x_{e 2}-x_{e 1}\right)}{\dot{\mathrm{m}}_{i}^{2}\left(x_{i 1}-x_{e 1}\right)}$,

$\frac{\partial \eta}{\partial \mathrm{x}_{\mathrm{e} 2}}=\frac{m_{e}}{\dot{\mathrm{m}}_{i}\left(x_{i 1}-x_{e 1}\right)}, \frac{\partial \eta}{\partial \mathrm{x}_{\mathrm{e} 1}}=\frac{\dot{\mathrm{m}}_{e}\left(x_{e 2}-x_{i 1}\right)}{\dot{\mathrm{m}}_{i}\left(x_{i 1}-x_{e 1}\right)^{2}}$

and $\frac{\partial \eta}{\partial x_{i 1}}=-\frac{\dot{\mathrm{m}}_{e}\left(x_{e 2}-x_{e 1}\right)}{\dot{\mathrm{m}}_{i}\left(x_{i 1}-x_{e 1}\right)^{2}}$ are partial derivations of equation (11) and $\delta_{\dot{\mathrm{m}}_{e}}, \delta_{\dot{\mathrm{m}}_{i}}, \delta_{\mathrm{x}_{i 1}}, \delta_{\mathrm{x}_{e 1}}, \delta_{\mathrm{x}_{e 2}}$ are total uncertainties of separated quantities.

From here, we can see that in order to get the uncertainty of moister transfer we have to calculate uncertainties of mass flows and specific humidity. Before that, we need to know other related uncertainties. Calculations of these uncertainties will be shown in following text.

\section{Temperatures, relative humidity, atmospheric pressure}

Device Almeno and sensors FHAD36R were used to monitor and $\log$ relative humidity, temperature of airflows and barometric pressure. Sensor FHAD36R has accuracy $\pm 1 \%$ of relative humidity and long-term stability is lower than $1 \%$ of relative humidity [11].

Temperatures, relative humidity, atmospheric pressure are three quantities that we got directly from raw data. Their uncertainties then can be derived using equations (8) and (9).

\section{Saturation pressure of water vapour of moist air}

Quality $p "{ }_{p}$ can be calculated using equation specified in [6], and its uncertainty is function of temperature of the air. We can obtain this value by using partial derivation with the variable of temperature

$$
\frac{\partial p^{\prime \prime} p}{\partial t}=-\frac{4044.2}{(235.6+t)^{2}} e^{23.58-\frac{4044.2}{235.6+t}}
$$

Then uncertainty of $p^{\prime \prime}$ is then

$$
\delta_{p_{p} p}=\frac{\partial p^{\prime \prime}}{\partial t} \delta t
$$

Specific humidity $x$ is given by equation

$$
x=0.622 \frac{\varphi p_{p}}{p_{b}+\varphi p_{p}},
$$

where $\varphi$ is relative humidity. The uncertainty of specific humidity is derived as

$$
=\sqrt{\left(\frac{\partial x}{\partial \varphi} \cdot \delta_{\varphi}\right)^{2}+\left(\frac{\partial x}{p_{b}} \cdot \delta_{p_{b}}\right)^{2}+\left(\frac{\partial x}{p_{p}{ }_{p}} \cdot \delta_{\left.p_{p}\right)}\right)^{2}},
$$

\section{Volumetric flow}

It is important to maintain flow rates in two flows at given values during measurement. We use two variable area meters (rotameters) to monitor mass flows; their factory's uncertainty is $\pm 3 \%$. We also verified their accuracy by using another high precise volume meter. We absorbed that, these rotameters work well at flow rates from $400 \div 1600 \mathrm{l} / \mathrm{h}$ but the position of rotating float became unstable. In addition, pressure of airflow before and after passing the float differed. The actual volumetric flow is given by read nominal volumetric flow and by both, nominal and actual densities by relation

$$
\dot{V}_{m}=\dot{V}_{n} \cdot \sqrt{\frac{\rho_{n}}{\rho_{m}}}
$$

where $\dot{V}_{\mathrm{n}}$ is the nominal value of flow, which is read on the scale of variable area meter, $\rho_{n}$ is nominal density and $\rho_{\mathrm{m}}$ is actual density of flowing air.

The uncertainty of volumetric flow is

$$
\delta_{\dot{V}_{m}}=\sqrt{\begin{array}{c}
\left(\frac{\partial \dot{V}_{m}}{\partial \dot{V}_{n}} \cdot \delta_{\dot{V}_{n}}\right)^{2}+\left(\frac{\partial \dot{V}_{m}}{\partial \rho_{n}} \cdot \delta_{\rho_{n}}\right)^{2}+ \\
+\left(\frac{\partial \dot{V}_{m}}{\partial \rho_{m}} \cdot \delta_{\rho_{m}}\right)^{2}
\end{array}},
$$




\section{Mass flows}

Relation between mass flow rate of dry air $\dot{m}_{\text {dry air }}$ and measured volumetric flow rate $\dot{V}_{m}$ is

$$
\dot{m}_{\text {dryair }}=\frac{\rho \dot{V}_{m}}{1+x} .
$$

The uncertainty of mass flow rate is

$$
=\sqrt{\left(\frac{\partial \dot{m}}{\partial \dot{V}} \cdot \delta_{\dot{V}}\right)^{2}+\left(\frac{\partial \dot{m}}{\partial \rho} \cdot \delta_{\rho}\right)^{2}+\left(\frac{\partial \dot{m}}{\partial x} \cdot \delta_{x}\right)^{2}},
$$

where partial derivatives are

$$
\begin{aligned}
& \frac{\partial \dot{m}}{\partial \dot{V}}=\frac{\rho}{1+x} \\
& \frac{\partial \dot{m}}{\partial \rho}=\frac{\dot{V}}{1+x}
\end{aligned}
$$

and

$$
\frac{\partial \dot{m}}{\partial x}=-\frac{\rho \dot{V}}{1+x^{2}}
$$

\section{Density of moist air}

Density of moist air according to [9] is calculated from equation of state of ideal gas as a mixture of dry air and water vapour

$$
\rho_{\text {moist }}=\frac{p_{\text {dryair }}}{r_{\text {dryair }} T_{0}}+\frac{p_{\text {moist }}}{r_{\text {moist }} T_{0}} .
$$

With room condition we can use bellow equation [9]

$$
\rho_{\text {moist }}=\frac{1.316 \cdot 10^{-3}}{T_{0}}\left(2.56 \cdot p+\varphi \cdot p_{m}^{\prime \prime}\right) \text {. }
$$

In the same way, we calculated the density's uncertainty of moist air.

\section{Results}

\section{Uncertainties' source analysis}

First, we evaluate the uncertainty of the moisture transfer. Effectiveness and its uncertainty at the flow rate $200 \mathrm{l} / \mathrm{h}$ is $(63.89 \pm 1.53) \%$. Contributions of uncertainty of individual quantities to resulting uncertainty of effectiveness are in table 1. Measurement of mass flow rates contributes to the resulting relative uncertainty by $2.4 \%$ each. The rest uncertainty is caused by measuring values of specific humidity and the highest contributions of $51.8 \%$ and $41.8 \%$ are given by measurement of $x_{i 1}$ and $\mathrm{x}_{\mathrm{e} 2}$ respectively, while influence of $\mathrm{x}_{\mathrm{e} 1}$ is negligible and of $2 \%$.

That means the uncertainties of specific humidity have the dominant impact on uncertainty of measuring moisture transfer. Analysis components of uncertainty of measuring specific humidity unveils the dominant causes (table 2.). Which are the uncertainty of relative humidity, contributes $28.6 \%$ and the uncertainty of partial saturated moist air, contributes $71.4 \%$. In other hand, partial saturated moist air is the function of only temperature (equation14.). Therefore, reducing the uncertainty of measuring flows' temperature and relative humidity will help reducing uncertainty of efficiency measurement.

\section{Moisture transfer's efficiency of membranes}

Figure 5 shows the curve of effectiveness of presented membrane: the lower the velocity of airflow is, the greater is the effectiveness. It is clear that the velocity of airflows has great impact on the value of effectiveness. Along with that, graph also presents the uncertainty of measurement. The greater efficiency is, the bigger uncertainties values are.

\section{Error and uncertainty of measurement}

Error in this paper presents the difference between moisture absorbed and released by membrane. The measurement was carried out when the moisture absorption and release process of membrane are stable. However, it is difficult to prove that these processes are parallel, especially due to accumulation of water inside membranes.

While uncertainty of measurement is relevant, the error values is considerable (table 4). This can be expressed in term of "high precision but less accuracy", means that measured values are close to each other but far from the "true" or acceptable values, read [12]. For this particulate sample, the error values are fairly small but for some others the errors are greater. Empirically, we noticed that the error values has strong connection to the ability of moisture transfer of membrane - if a membrane has a high moisture transfer effectiveness, then low measuring error is obtained and vice versa (with other conditions remain unchanged). 
Table 1: Individual contributions in the uncertainty budget of effectiveness measuring (resulting of equation 12)

\begin{tabular}{|l|c|c|c|c|c|}
\hline Function & $\left(\frac{\partial \eta_{m}}{\partial \mathrm{m}_{\mathrm{i}}} \delta_{\mathrm{m}_{i}}\right)^{2}$ & $\left(\frac{\partial \eta_{m}}{\partial \mathrm{m}_{e}} \delta_{\mathrm{m}_{e}}\right)^{2}$ & $\left(\frac{\partial \eta_{m}}{\partial \mathrm{x}_{\mathrm{i} 1}} \delta_{\mathrm{x}_{i 1}}\right)^{2}$ & $\left(\frac{\partial \eta_{m}}{\partial \mathrm{x}_{\mathrm{e} 1}} \delta_{\mathrm{x}_{e 1}}\right)^{2}$ & $\left(\frac{\partial \eta_{m}}{\partial \mathrm{x}_{\mathrm{e} 2}} \delta_{\mathrm{x}_{e 2}}\right)^{2}$ \\
\hline Value & 0.0004 & 0.0004 & $\mathbf{0 . 0 0 7 9}$ & $\mathbf{0 . 0 0 0 3}$ & $\mathbf{0 . 0 0 6 4}$ \\
\hline Contribution & $2.4 \%$ & $2.4 \%$ & $\mathbf{5 1 . 4 \%}$ & $2 \%$ & $\mathbf{4 1 . 8 \%}$ \\
\hline
\end{tabular}

Table 2: Contributions to uncertainty of specific humidity measuring

\begin{tabular}{|l|c|c|c|}
\hline Function & $\left(\frac{\partial x}{p_{b}} \cdot \delta_{p_{b}}\right)^{2}$ & $\left(\frac{\partial x}{p_{p}} \cdot \delta_{p_{p} p}\right)^{2}$ & $\left(\frac{\partial x}{\partial \varphi} \cdot \delta_{\varphi}\right)^{2}$ \\
\hline Value & $1.5 \cdot 10^{-13}$ & $3.7 \cdot 10^{-9}$ & $1.5 \cdot 10^{-9}$ \\
\hline Contribution & $2.8 \cdot 10^{-3} \%$ & $\mathbf{7 1 . 4 \%}$ & $\mathbf{2 8 . 6 \%}$ \\
\hline
\end{tabular}

Table 3: Correlation between absolute uncertainty and flow's velocity

\begin{tabular}{|l|c|c|c|c|c|c|c|c|c|c|}
\hline $\begin{array}{l}\text { Flow's velocity } \\
\text { m/s }\end{array}$ & 0.26 & 0.52 & 0.78 & 1.03 & 1.29 & 1.55 & 1.81 & 2.07 & 2.33 & 2.58 \\
\hline Efficiency \% & 63.89 & 45.93 & 39.38 & 33.72 & 30.07 & 26.88 & 24.75 & 23.62 & 22.62 & 21.64 \\
\hline Uncertainty \% & 1.53 & 1.39 & 1.15 & 0.98 & 0.82 & 0.73 & 0.63 & 0.55 & 0.48 & 0.43 \\
\hline Error \% & 4.29 & 7.33 & 6.44 & 5.21 & 5.85 & 5.37 & 5.35 & 4.99 & 4.86 & 3.4 \\
\hline
\end{tabular}

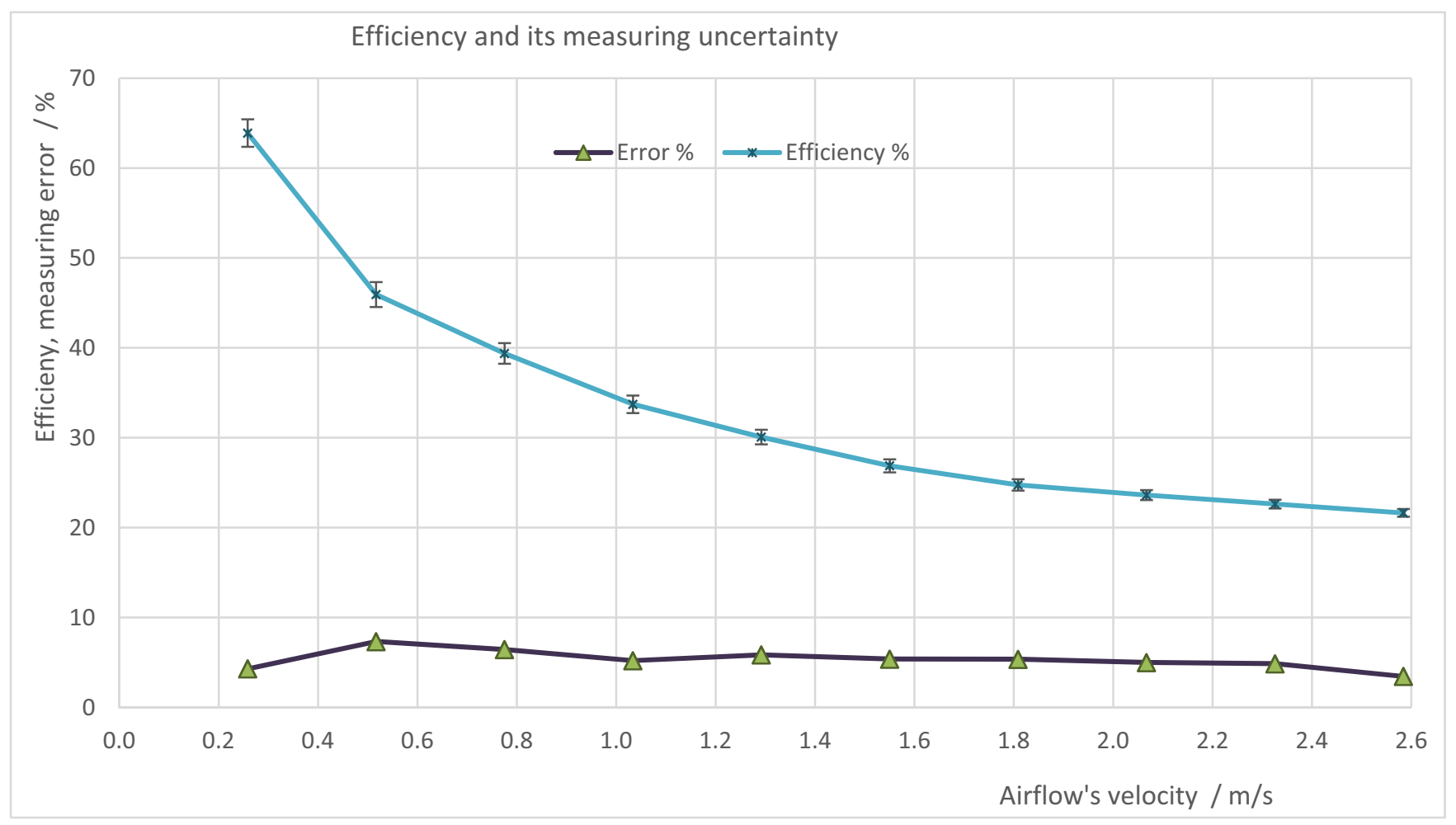

Figure 5: Moisture transfer's efficiency and measuring error 


\section{Discussion and conclusion}

By analysis measuring uncertainties we unveiled that the uncertainty of sensors FHAD36R cause the imprecision. Actually, according to manufacturer, those sensors are very accuracy and precision. We may need to perform a calibration on them, or try other devices to compare results.

Although the measuring error was acceptable, there are still possible ways to improve measuring procedure. Based on experiences during this experimental investigation, we would like to consider improving our testing device. First, we need to log mass flow rates continuously into PC during the entire testing process by precise digital mass flow meters. By doing this, we can reduce uncertainty of flow rates.

The change of inlet relative humidity due to change in airflow velocity is carried out in table 4 . Upper row is the velocity of airflow $(\mathrm{m} / \mathrm{s})$ and the lower row is the corresponding relative humidity $(\%)$. This phenomenon makes the evaluation of effectiveness less impact. Ideally, humidity of airflows should be stable and constant across for all flow velocities and all tested membranes.

Table 4: Variation of inlet relative humidity relevant to airflow's velocity

\begin{tabular}{|l|l|l|l|l|l|l|l|l|l|}
\hline $\begin{array}{l}\text { Velocity of } \\
\text { flow (m/s) }\end{array}$ & 0. & 0. & 0. & 1. & 1. & 1. & 1. & 2. & 2. \\
\hline RH $(\%)$ & 63 & 52 & 78 & 03 & 29 & 55 & 81 & 07 & 33 \\
\hline & .0 & .6 & .9 & 67 & 69 & 71 & 73 & 76 & 76 \\
& & & & & .4 & .0 & .9 & .0 & .3 \\
\hline
\end{tabular}

Second, we will add a soft-seat valve right after the flap (5) to control more precisely the mass flow rate of air entering the vaporizer or design and use more convenient vaporizer.

Third it would be beneficial to use different approach to specify partial pressure of saturated vapour than equation (6).

Ambient temperature should be maintained unchanged during measurement (sometime lab's temperature changed significantly during a single measurement).

\section{Acknowledgment}

We gratefully acknowledge the support of the Student Grant Agency of the Technical University of Liberec (project no. 21124).

\section{References}

[1] S. M. Aarnes, "Membrane Based Heat Exchanger.," 2012.

[2] L. Z. Zhang and J. L. Niu, "Effectiveness Correlations for Heat and Moisture Transfer Processes in an Enthalpy Exchanger With Membrane Cores," J. Heat Transfer, vol. 124, no. 5, p. 922, 2002.

[3] W. Yaïci, M. Ghorab, and E. Entchev, "Numerical analysis of heat and energy recovery ventilators performance based on CFD for detailed design," Appl. Therm. Eng., vol. 51, no. 1-2, pp. 770-780, 2013.

[4] Z.-X. Li, T.-S. Zhong, J.-L. Niu, F. Xiao, and L.Z. Zhang, "Conjugate heat and mass transfer in a total heat exchanger with cross-corrugated triangular ducts and one-step made asymmetric membranes," Int. J. Heat Mass Transf., vol. 84, pp. 390-400, May 2015.

[5] L. Z. Zhang and J. L. Niu, "Effectiveness Correlations for Heat and Moisture Transfer Processes in an Enthalpy Exchanger With Membrane Cores," J. Heat Transfer, vol. 124, no. 5, p. 922, 2002.

[6] V. Dvořák. P. Novotý, N. Van Vu, "Measurement of Moisture Transport in the Membrane-Based Enthalpy Exchanger," EFM, 2013.

[7] L. Z. Zhang, "Heat and mass transfer in a quasicounter flow membrane-based total heat exchanger," Int. J. Heat Mass Transf., vol. 53, no. 23-24, pp. 5499-5508, 2010.

[8] N. Van Vu, "Evaluation properties of membranes for enthalpy heat exchanger," Technical univesity of Liberec, 2015.

[9] J. Chyský, K. Hemzal a kol., Větrání a klimatizace, Technický průvodce. Praha: SNTL, 1993.

[10] J. Sulc, V. Dvorak, “Technická měření - návod k cvicení,” Liberec: Vysokoškolský podnik, 2005, p. 60 .

[11] Ahlborn, "Sensor fhad36r," 2012. .

[12] J. R. Taylor, An Introduction to Error Analysis: The Study of Uncertainties in Physical Measurements , $2 d$ Edition. University Science Books, 1997. 